\title{
Ten Commandments of Safe and Optimum Neck Dissections for Cancer
}

\author{
Chintamani $^{1}$
}

Published online: 5 June 2015

(C) Association of Surgeons of India 2015

Neck nodes are the best predictors of outcome in head and neck cancers, and the survival drops by $50 \%$ in node-positive necks. Neck dissections are therefore an essential aspect of management of head and neck cancers. Most surgeons dedicated to head and neck surgery must have a thorough understanding of surgical anatomy of this region and steps essential for performing this surgery optimally and safely.

\section{History and Evolution of Neck Dissections [1-3]}

The first neck dissection was performed by GW Crile in the year 1906 (he preserved the spinal accessory nerve for some reason). Subsequently, various other surgeons like Martin made it more and more radical and insisted on removal of spinal accessory nerve to make it optimum. The better understanding of the lymphatic drainage of head and neck region by Rouviere et al. made it possible to predict the pattern of lymphatic drainage in a cancer involving a particular region, and neck dissections thus became more and more conservative/ selective. Bocca provided the first classification of modified radical neck dissections (MRND), and preservation of any one structure made it MRND I, while if all the three extralymphatic structures were preserved, it was called MRND III or functional neck dissections (FND).

Chintamani

drchintamani7@gmail.com

Vardhman Mahavir Medical College, Safdarjang Hospital, New Delhi, India
These commandments are by no means the only way to perform this surgery but "one correct way" of doing it. There is no rigidity in these steps, and these are merely recommendations to achieve an optimal outcome safely.

\section{Commandment 1. Not to Operate by Clock}

A surgeon operating by clock has no business to be in this region that has too many vital structures packed closely together. A thorough understanding of surgical anatomy is mandatory to avoid lethal injuries. Fortunately, on most occasions, the surgical anatomy is by and large fixed, and it amounts to cutting on the dotted line. One has to only follow the "holy bloodless planes" in order to achieve an optimal clearance.

\section{Commandment 2. Postioning Correctly and Making the Right Incision}

The classical position for most head and neck surgeries is reverse Trendelenberg with extension at the neck to have maximum access. The head is turned away to the opposite side in order to expose all the triangles of the neck adequately (Figs. 1, 2). Raising the head end of the table by $15^{\circ}$ reduces venous congestion in the region but also adds to the risk of air embolism by creating negative pressure in the veins. The recommended method to avoid this catastrophe is to ligate these veins in continuity, i.e. ligating before cutting them (Table 1).

There are numerous incisions for neck dissections, but the most common and the one used by the author is "modified Schobinger's incision". The incision has two limbs, one horizontal and the other one comes down vertically as a lazy "s" (in order to prevent scar contracture later). The horizontal limb typically lies at least about $2 \mathrm{~cm}$ below the mandible to avoid 


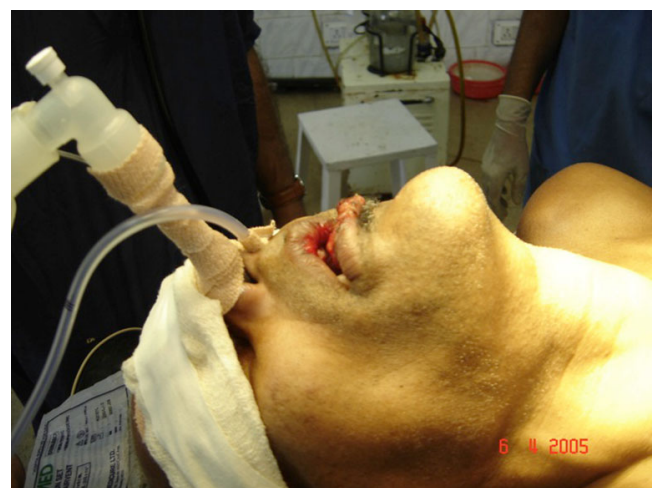

Fig. 1 Positioning: reverse Trendelenburg with neck extended and turned to the opposite side exposes all triangles optimally

injury to the ramus mandibularis, and the vertical limb starts at a point that is at least $2 \mathrm{~cm}$ behind the point where the carotid pulsations are felt. This is to avoid the trisection from lying directly over the carotids (in the event of the dehiscence of incision at this trisection, the carotids may lie exposed leading to a blow out, especially following radiotherapy). This also allows the posterior flap to be as short as possible as it is devoid of platysma, which is vital for the survival of these flaps. There are some other popular incisions in use like the "Mcfee incision" which is in the form of a step ladder [it is particularly useful in the irradiated necks where flap failure rates are "expectedly" higher owing to poor blood supply]. The "utility incision" is frequently used when surgery is being performed for thyroid cancer, and neck dissection is also contemplated.

\section{Commandment 3: Raising the Flaps Adequately and Finding the "Holy Planes" and the Ramus Mandibularis}

The next step is to raise the sub-platysmal flaps (anterior and posterior). As the access to all parts is limited, one has to rely on well-raised platysmal flaps to reach all end points of dis-

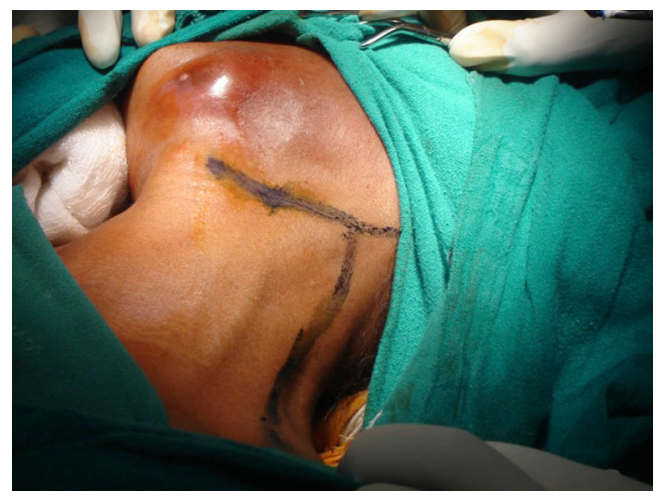

Fig. 2 Incision: modified Schobinger's incision. The trisection at the junction of the vertical and horizontal limbs must be placed posterior to the carotid pulsations
Table 1 The commandments

\section{Thou shall}

1.Not operate by clock!!

2.Position correctly and make right incision

3.Raise the flaps adequately and find the "holy planes", ramus mandibularis

4.Not look for aberrations as anatomy in the head and neck "generally" predictable

5.Search for the anatomical "lighthouses"-Erbs' point, phrenic nerve, mmohyoid, facial vein etc.

6.Identify internal and external carotid vessels, hypoglossal nerve

7.Always find "facio-jugular lymph node"

8. Find the digastric to reach level I (b) and I (a)

9.Aim to preserve extra-lymphatic structures [SAN, IJV, SCM] but not at the cost of oncological safety

10.Shall preserve at least one side IJV.

section. While raising the superior flap, one has to be very cautious in order to preserve the marginal mandibular nerve or "ramus mandibularis" which is a branch of facial nerve that supplies the angle of mouth. The morbidity following injury to this nerve is severe in terms of poor oral competence, drooling of saliva and difficulty in speech. There are various techniques to protect this nerve, and the author likes to use the "Hayes Martin" technique that involves dissecting the common facial vein in the neck and transecting after ligating it. The upper cut end is hitched up to get the nerve out of harm's way (Figs. 3, 4 and 5).

\section{Commandment 4: Not to Look for Aberrations and Sticking to the Established Plan}

Surgery in this region amounts to cutting on the dotted line as the anatomy is usually fixed and aberrations are rare (and are often only in the mind of the surgeon). One has to be absolutely thorough with the surgical anatomy in order to avoid bleeding and/or injury to vital structures. The dissection now proceeds in a predictable way in a bloodless field demonstrating each structure to the assistants. This is made possible by fol-

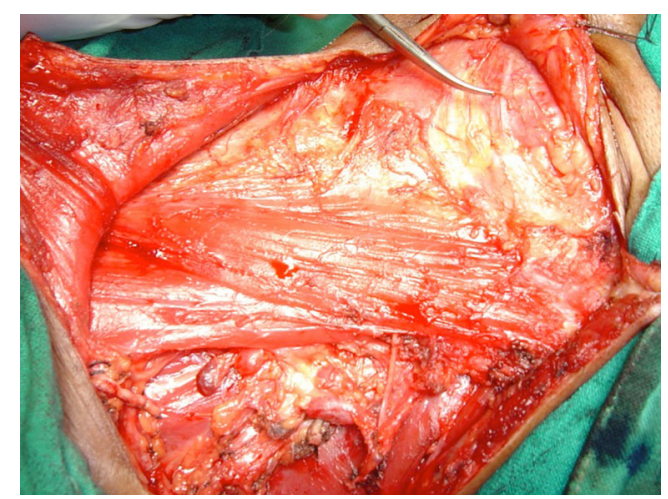

Fig. 3 Flaps: the ramus mandibularis and the spinal accessory nerves may be seen 

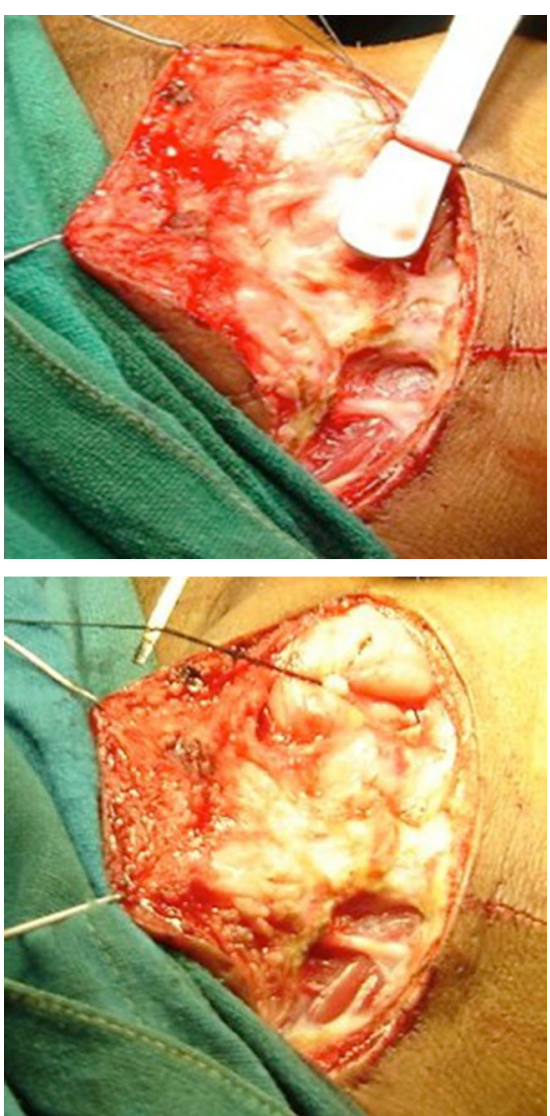

Fig. 4 \& 5 Hayes Martin technique of safeguarding the ramus mandibularis. The common facial vein is dissected, ligated and transected, and the upper end is hitched out of harm's way

lowing the basic surgical principles of neat dissection in a bloodless field and sticking to "God's planes" from level V or the posterior triangle upwards and medially dissecting levels IV, III, II, Ib and Ia and preferably removing the primary lesion en bloc to conclude the surgery. Some surgeons prefer to take an antero-posterior (i.e. level I to level V) approach, which is equally good.

\section{Commandment 5: Searching for the Anatomical Lighthouses}

Each step is taken in a direction to reach an end point or what the author would like to call a "lighthouse". These lighthouses make the entire dissection fairly predictable and reproducible ensuring an optimum clearance each time.

Lighthouses in the posterior triangle:

- Erb's point: where the greater auricular nerve winds around the sternocleidomastoid muscle in the posterior triangle. The spinal accessory nerve (SAN) enters the posterior triangle approximately $2 \mathrm{~cm}$ superior to this point (Fig. 6).

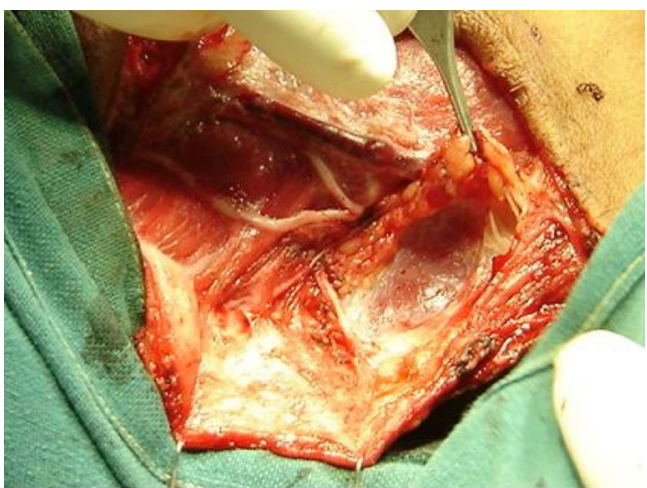

Fig. 6 Erbs's point: the point where the greater auricular nerve winds around the sternocleidomastoid muscle can be seen. The spinal accessory nerve can be seen entering the posterior triangle approximately $2 \mathrm{~cm}$ superior to this point

- Safety layer: this prevertebral layer of deep fascia that covers the phrenic nerve and the brachial plexus is also called the "Holy layer". If not infiltrated by cancer itself, one can avoid injury to the phrenic nerve and the brachial plexus by staying superficial to it during dissection of level V or the posterior triangle (Figs. 6 and 7).

- Phrenic nerve: this is the only structure that traverses lateral to medial in the neck, all other structures travel either medial to lateral or from above downwards (Fig. 7). By dissecting and staying superficial to this layer, injury to the brachial plexus and the phrenic nerve is avoided, and unless directly involved by the disease, all these structures must be protected (Fig. 7).

- Omohyoid (Figs. 8 and 9): This muscle serves as a very important lighthouse that traverses from posterior to anterior triangle crossing the internal jugular vein (IJV). Many surgeons including the author would retract rather than cut this muscle and look for a level IV lymph node (rather than routinely dissect level IV as there is a risk of injury to

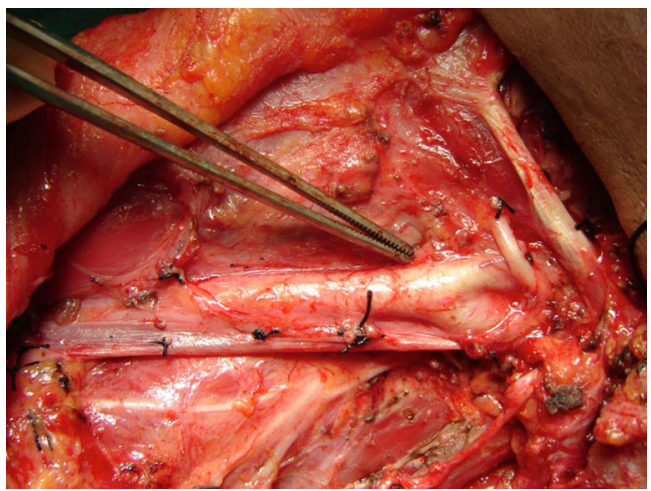

Fig. 7 The internal and external carotid arteries, phrenic nerve, SAN, and hypoglossal nerve can be seen. The external carotid artery gives off the superior thyroid artery as its first branch and lies internally. The hypoglossal nerve crossing in between the two carotids approximately $2 \mathrm{~cm}$ superior to the bifurcation of the common carotid artery may be seen 


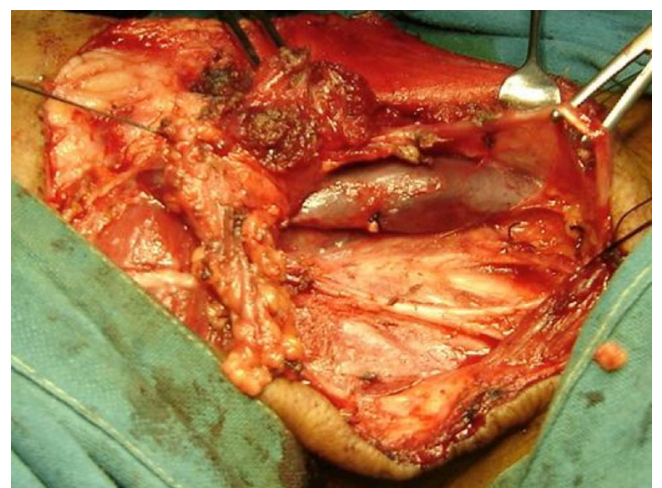

Fig. 8 Omohyoid muscle and the floor of posterior triangle

vital structures like a thoracic duct on the left side). Moreover, level IV is not very commonly involved in most head and neck cancers, routine level IV dissection is therefore not recommended except in scenarios like cancers of tongue where skip metastases to this level are known or poor grade cancers.

Lighthouses in the anterior triangle:

The dissection proceeds along the IJV, and carotid sheath is routinely removed along with levels II, III and IV nodes. This step exposes the common carotid artery and the vagus nerve that lies between the IJV and the common carotid artery.

- Facial vein (Figs. 10, 11 and 12): this is the last tributary of the IJV that would need to be ligated, and this also guides one to the facio-jugular node (level IIa), which may serve as the "sentinel lymph node" in certain oral cavity lesions. The vein crosses the common carotid artery to drain in to the IJV. This last tributary may need to be ligated before the IJV is ligated and transected as close to the skull base as possible if one is performing the classical radical neck dissection (RND).

- Posterior belly of digastric: This lighthouse can be used as a guide to move towards level I after the dissection at level

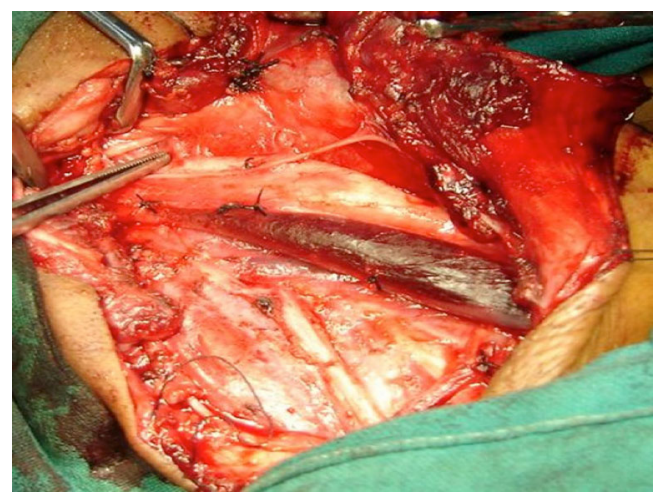

Fig. 9 The external carotid artery gives off branches in the neck and lies internally
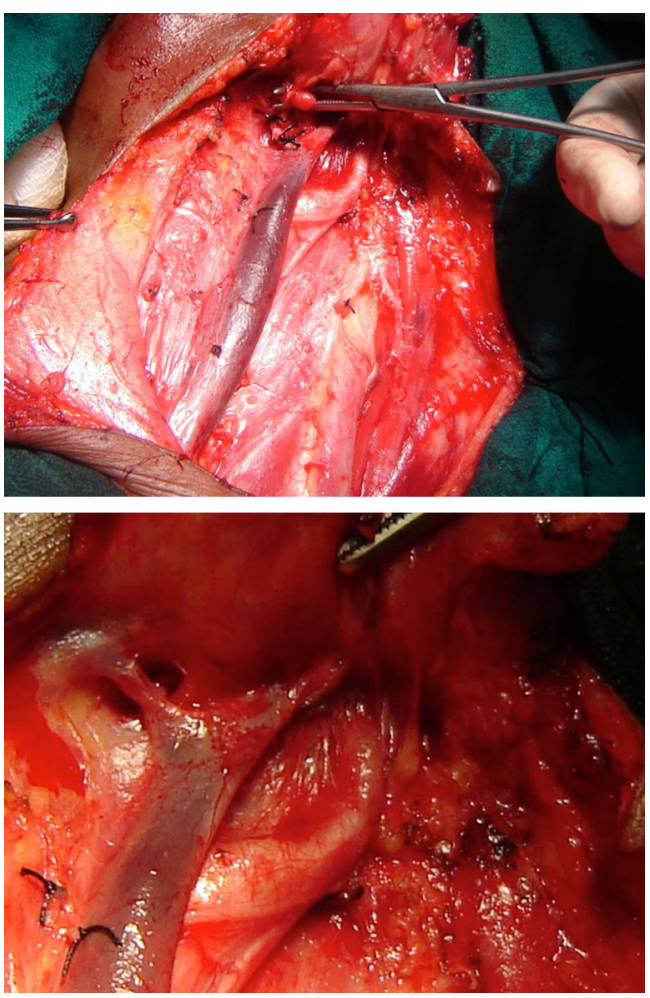

Fig. 10 and 11 The facio-jugular node lies in the angle between the internal jugular vein and its last tributary, the common facial vein. It is considered by some as the sentinel node for the cancers of buccal mucosa

II is complete (Fig. 13). The hypoglossal nerve crosses in to the submandibular triangle or level I (b) under this muscle.

\section{Commandment 6: Identify Internal and External Carotid Vessels and the Hypoglossal Nerve}

As mentioned above, the carotid sheath is opened, and levels II and III are dissected along with the sheath. The external

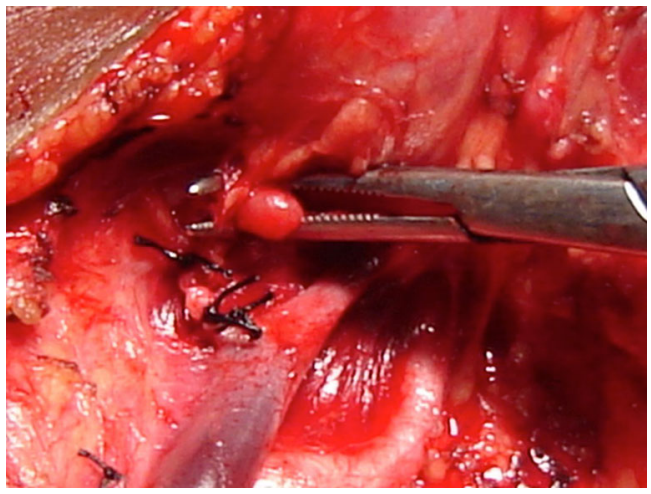

Fig. 12 The facio-jugular node being dissected out 


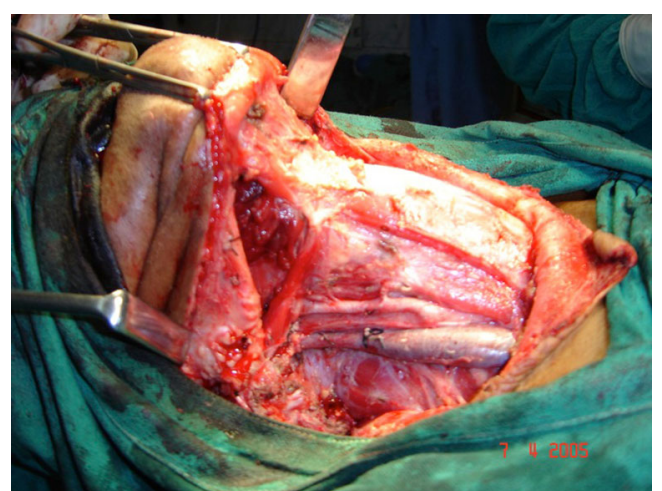

Fig. 13 Bilateral neck dissection completed. The digastric bellies serve as important lighthouses to reach and clear level I (a) and I (b). The hypoglossal nerve can be seen crossing in between the internal and external carotid arteries approximately $2 \mathrm{~cm}$ superior to the bifurcation of common carotid artery

carotid artery is actually "internal" in anatomical location and gives off branches in the neck, the first one being the "superior thyroid artery". About $2 \mathrm{~cm}$ superior to the bifurcation of the "common carotid artery", the "hypoglossal nerve" can be seen crossing the carotids and ascending towards level I (Fig. 7).

[While dissecting close to the carotid bifurcation, it is mandatory to alert the anesthesiologist, as there may be bradycardia or even cardiac arrest due to stimulation of baroreceptors that can be corrected/prevented by infiltrating $1 \%$ lignocaine].

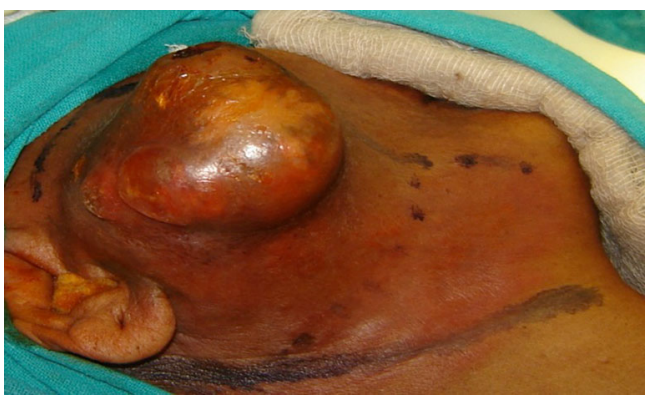

Fig 18 A 50-year-old lady with carcinoma parotid with infiltration of skin and facial nerve involvement being planned for radical parotidectomy and right-sided MRND type III

\section{Commandment 7: Always Find the "Facio-jugular Lymph Node"}

The dissection now proceeds upwards and medially, and the facio-jugular lymph node (or level II (a)) is dissected. The common facial vein may be ligated and transected at this point, and one may proceed along this vein to reach level I or one may also trace the posterior belly of digastric to reach level I as mentioned previously (Fig. 12).

\section{Commandment 8: Find the Digastric to Reach Level I (b) and I (a)}

One may now dissect along the posterior belly of the digastric muscle to reach level I (Fig. 13). The facial vessels (artery and
Figs. 14, 15, 16 and 17 A fungating carcinoma involving the classical site for an Indian oral cancer (lower gingiva-buccal sulcus) and extending on to either side of midline. A bilateral neck dissection along with extensive composite resection of the lesion has been performed. IJV must be preserved at least on one side. The reconstruction being performed using occipital artery-based axial flap
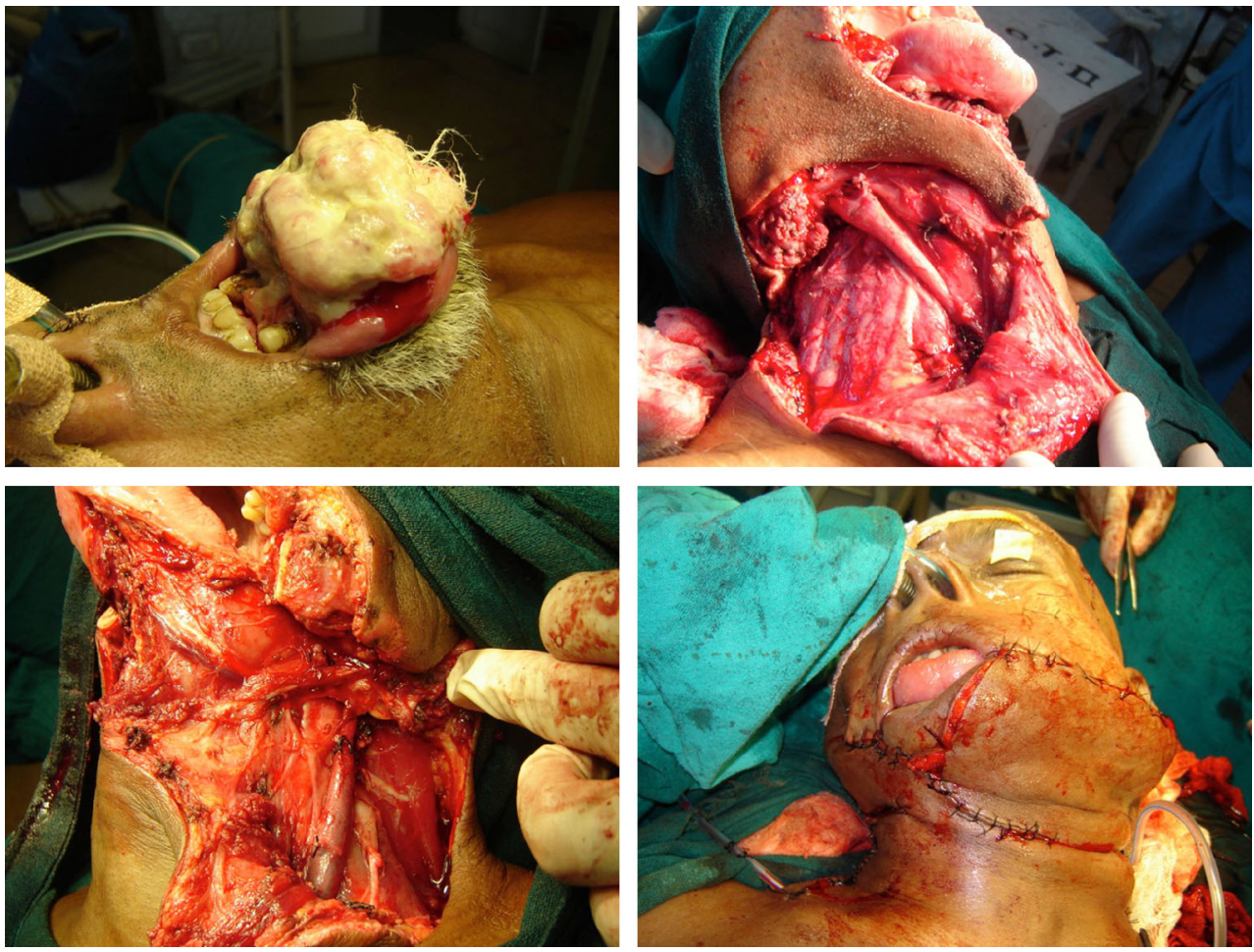

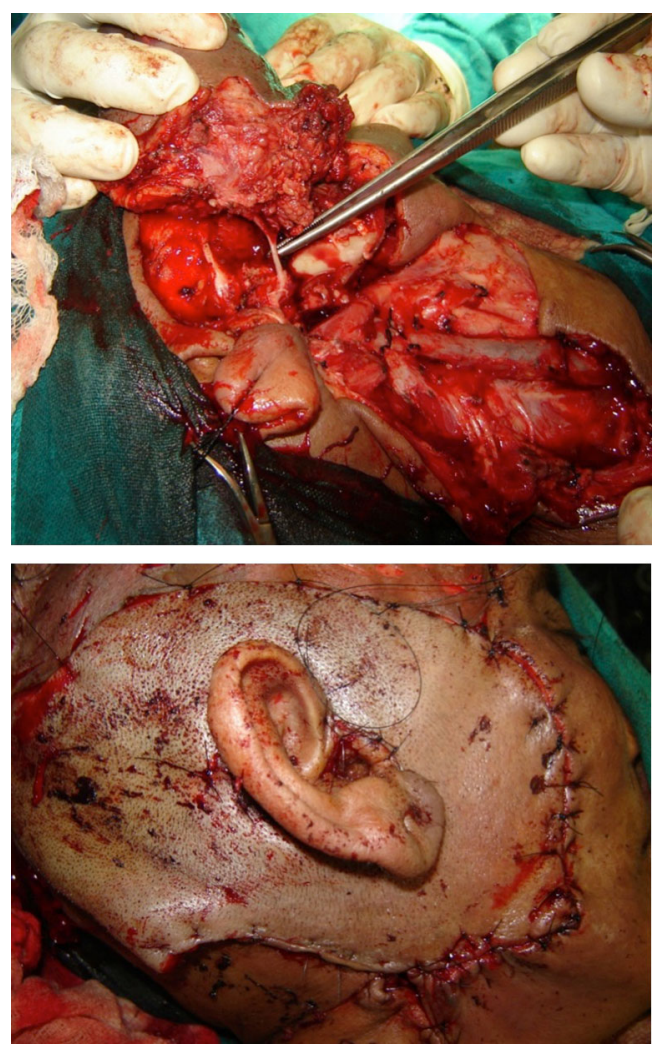

Figs. 19 and 20 The radical parotidectomy in progress, the facial nerve is resected flush at the point of exit from the foramina. The MRND III and reconstruction performed using a local axial transposition flap based on occipital artery

vein) both would need to be ligated and transected or may be preserved if not directly involved to utilize them as recipient vessels for a "free flap".

\section{Commandment 9: Aim to Preserve Extra-Lymphatic Structures [SAN, IJV, SCM] but not at the Cost of Oncological Safety}

The classical radical neck dissection (RND) involves removal of lymph nodes from levels I to $\mathrm{V}$ along with three extra-

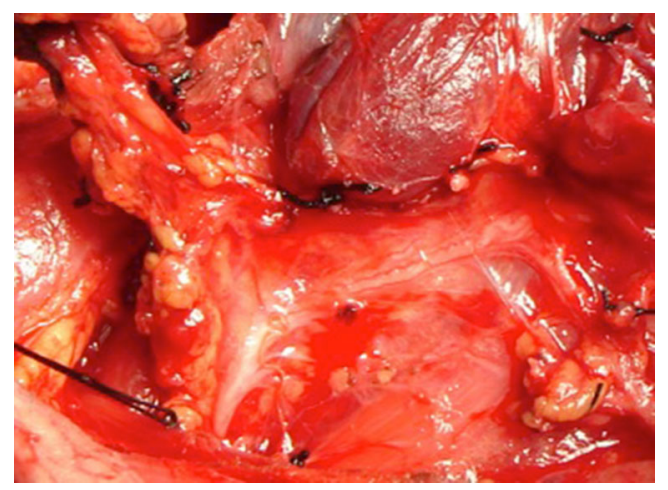

Fig. 21 Central compartment neck dissection (level VI) for medullary carcinoma thyroid in progress. The lymphatics are dissected in the fossa along the recurrent laryngeal nerve as it enters the neck

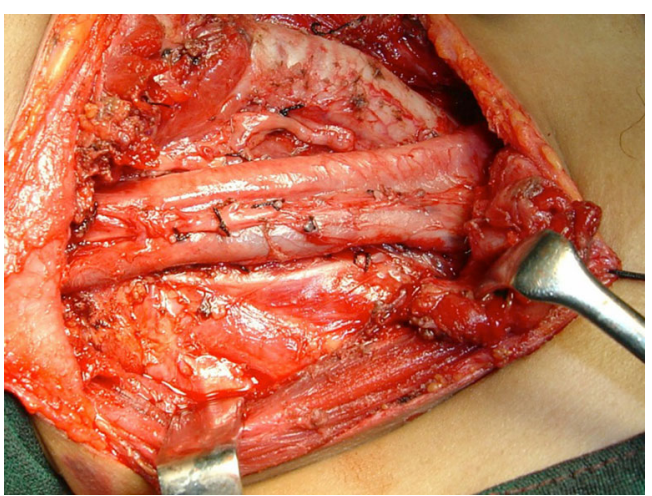

Fig. 22 In certain cases (like in this case), the level VI dissection may have to combine with MRND III using the utility incision. The sternocleidomastoid muscle may be retracted to dissect level V. The spinal accessory nerve, common carotid, vagus nerve and IJV (all preserved) may be seen. The preservation of all parathyroids is a challenge but is mandatory besides preservation of recurrent laryngeal and external laryngeal nerves on both sides

lymphatic structures spinal accessory nerve (SAN), internal jugular vein (IJV) and sternocleidomastoid muscle (SCM). Over the years, it has been extensively studied that these structures may be preserved unless directly involved by the disease. Depending on the number of structures preserved, it may be called as modified radical neck dissection (MRND I) if only one is preserved or (MRND II) if two are preserved or MRND III if three structures are preserved. In the order of importance, SAN gets a preference over IJV, and SCM preservation is the last but not the least priority.

\section{Commandment 10: Shall Preserve the Internal Jugular Vein at Least on One Side}

If bilateral neck dissections are contemplated, IJV must be preserved at least on one side in order to avoid the morbidity of facial edema and high mortality (Figs. 14, 15, 16 and 17).

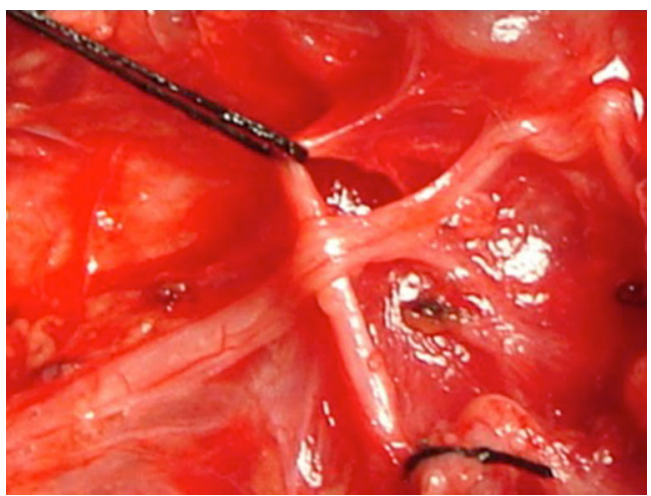

Fig. 23 The inferior thyroid artery (ITA) has been hooked on a sling to clear off all the lymphatics. The recurrent laryngeal nerve may be seen crossing through the capsular branches of ITA. The main trunk of ITA is never ligated unless injured; only the capsular branches may be ligated 


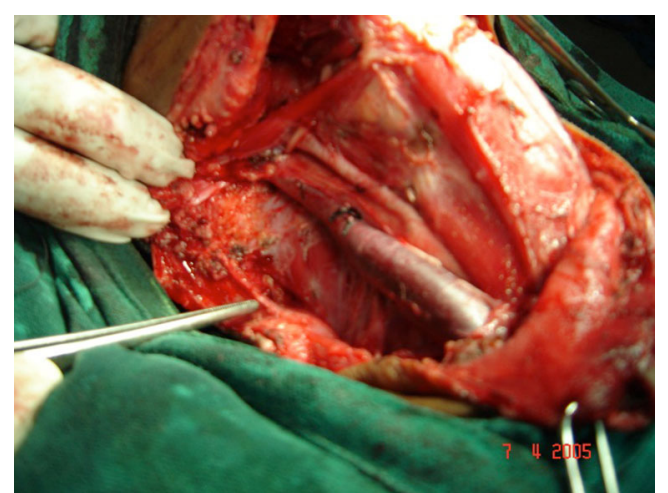

Fig. 24 Extended neck dissection completed. The levels I, II, III, IV, and VI have been removed in a medullary thyroid cancer. The spinal accessory is being pointed at. It actually amounts to MRND III and clearance of level VI. It is not usual to dissect level I in most thyroid cancers but may need to be done in positive necks

\section{Selective Neck Dissections}

There are occasions when neck dissections may not follow this conventional approach, and one may have to select and remove only limited neck node basins, based on the predicted map of lymphatic spread in the head and neck region. These are called selective neck dissections (SND), and one may simply mention the levels removed along with SND as a prefix. For example, in a classical supra-omohyoid neck dissection, levels I-III are removed; this may be described as SND I, II and III. Similarly, in certain scenarios like thyroid cancers, one may end up removing levels II, III and VI in view of the high probability of lymphatic spread to these regions, and the same may be described as SND II, III and VI [4].

\section{Extended Neck Dissections [1, 2, 4]}

Extended neck dissections may involve removal of more levels than the conventional levels $\mathrm{I}-\mathrm{V}$ in one particular case, e.g. in cancers of parotid (Figs. 18, 19, 20 and 21). In case of thyroid cancers, in addition to MRND III, level VI may also have to be removed (Figs. 22, 23 and 24).

\section{References}

1. Crile G (1906) Excision of cancer of the head and neck: with special reference to the plan of dissection based on 132 operations. JAMA 47:1780-1785

2. Martin H, Del Valle B, Ehrlich H, Cahan WG (1951) Neck dissection. Cancer 4(3):441-499

3. Shaha AR (2004) Radical neck dissection. Oper Tech Gen Surg 6(2): $72-82$

4. Chintamani Editorial (2010) "Ten Commandments" of safe and optimum thyroid surgery. Indian J Surg 72(6):421-426 